\title{
Algebraic Representation, Elimination and Expansion in Automated Geometric Theorem Proving
}

\author{
Hongbo $\mathrm{Li}^{1}{ }^{1}$
}

\begin{abstract}
Cayley algebra and bracket algebra are important approaches to invariant computing in projective and affine geometries, but there are some difficulties in doing algebraic computation. In this paper we show how the principle "breefs" - bracketoriented representation, elimination and expansion for factored and shortest results, can significantly simply algebraic computations. We present several typical examples on conics and make detailed discussions on the procedure of applying the principle to automated geometric theorem proving.
\end{abstract}

\section{Introduction}

Cayley algebra and bracket algebra are important approaches to invariant computations in projective geometry [1], [3], [7], [15], [16], [17], [18], [20], [21], [23], and have been used in automated theorem proving in projective geometry for over a decade [6], [9], [13], [14]. Recently, in a series of papers [10], [11], [12], the authors further applied the two algebras in theorem proving in projective and affine geometries, and developed some new techniques to overcome the following difficulties in the application.

1. Multiple algebraic representations. The simplest example is the constraint that six planar points $\mathbf{1}, \mathbf{2}, \mathbf{3}, \mathbf{4}, \mathbf{5}, \mathbf{6}$ are on the same conic. They must satisfy the following equality:

$$
\operatorname{conic}(\mathbf{1 2 3 4 5 6})=[\mathbf{1 3 5}][\mathbf{2 4 5}][\mathbf{1 2 6}][\mathbf{3 4 6}]-[\mathbf{1 2 5}][\mathbf{3 4 5}][\mathbf{1 3 6}][\mathbf{2 4 6}]=0 .
$$

Changing the order of the sequence, we get all together 15 different equalities:

$$
\begin{aligned}
& \operatorname{conic}(\mathbf{1 2 3 4 5 6})=0, \quad \operatorname{conic}(\mathbf{1 2 3 5 4 6})=0, \quad \operatorname{conic}(\mathbf{1 2 3 6 4 5})=0, \\
& \operatorname{conic}(\mathbf{1 2 4 3 5 6})=0, \quad \operatorname{conic}(\mathbf{1 2 4 5 3 6})=0, \quad \operatorname{conic}(\mathbf{1 2 4 6 3 5})=0, \\
& \operatorname{conic}(\mathbf{1 2 5 3 4 6})=0, \quad \operatorname{conic}(\mathbf{1 2 5 4 3 6})=0, \quad \operatorname{conic}(\mathbf{1 2 5 6 3 4})=0, \\
& \operatorname{conic}(\mathbf{1 2 6 3 4 5})=0, \quad \operatorname{conic}(\mathbf{1 2 6 4 3 5})=0, \quad \operatorname{conic}(\mathbf{1 2 6 5 3 4})=0, \\
& \operatorname{conic}(\mathbf{1 3 4 2 5 6})=0, \quad \operatorname{conic}(\mathbf{1 3 5 2 4 6})=0, \quad \operatorname{conic}(\mathbf{1 3 6 2 4 5})=0 \text {. }
\end{aligned}
$$

If the constraint occurs in the hypotheses, then which of the 15 equalities should be used? If it occurs in the conclusion, then which equality can lead to a simpler proof?

If there are more than 6 points on the conic, the number of equalities increases very fast.

Academy of Mathematics and System Sciences, Chinese Academy of Sciences, Beijing 100080, China 
2. Multiple elimination strategies. If there are multiple representations for the same constraint, there will be multiple elimination strategies.

Even if all representations are unique, and the elimination follows strictly the order of construction, there can still be multiple elimination strategies. For example, in bracket [ABC], all three points are intersections of lines, and all the lines have been constructed before the three points. Then the three points are constructed at the same time, and can be eliminated either one by one, or all at the same time, or in an intermediate fashion.

3. Multiple expansion results. In the projective plane, let $\mathbf{A}=\mathbf{1 2} \cap \mathbf{3 4}, \mathbf{B}=\mathbf{1}^{\prime} \mathbf{2}^{\prime} \cap$ $\mathbf{3}^{\prime} \mathbf{4}^{\prime}, \mathbf{1}^{\prime \prime} \mathbf{2}^{\prime \prime} \cap \mathbf{3}^{\prime \prime} \mathbf{4}^{\prime \prime}$. Eliminating $\mathrm{A}, \mathrm{B}, \mathrm{C}$ from bracket $[\mathrm{ABC}]$, we get

$$
\left[(12 \wedge 34)\left(1^{\prime} 2^{\prime} \wedge 3^{\prime} 4^{\prime}\right)\left(1^{\prime \prime} 2^{\prime \prime} \wedge 3^{\prime \prime} 4^{\prime \prime}\right)\right]
$$

Expanding a Cayley expression into a bracket polynomial is called Cayley expansion. Here (3) has 16,847 different expansions into bracket polynomials.

Then which one should we choose?

4. Multiple equivalent expressions. The bracket algebra is the quotient of the polynomial ring of brackets, by the ideal generated by Grassmann-Plücker syzygies. Because of this, a bracket polynomial has multiple different forms. It is natural to consider finding the "simpler" forms - factored or shortest ones.

This is a procedure of simplification. How to compute a bracket representation having the minimal number of tableaux is an open problem [17].

\section{From brackets to vector expressions - Cayley factorization.}

The multi-linear case is solved in [21]. The general case is an open problem.

Despite the difficulties, it is quite worthwhile and rewarding to consider using invariant algebras in automated geometric theorem proving, because amazing simplifications can be achieved.

In [10], a complete classification of factored expansions and binomial expansions of all Cayley expressions resulted from eliminating points in a bracket in $2 \mathrm{D}$ or $3 \mathrm{D}$ projective geometry is carried out. This solves the third problem for 2D and $3 \mathrm{D}$ cases.

In [11], various representations of conics and related geometric entities are established, together with their transformation properties. In this paper, the work is further extended to include more geometric constructions. This solves the third problem for the case of projective and affine conics.

The idea of batch elimination comes from Cayley expansion. In [10], the points constructed at the same time with the same fashion can be eliminated at the same time, and several techniques are developed to reduce a bracket polynomial to a factored or shortest form. In [11], the techniques are further developed to include points satisfying multiple nonlinear constraints. In [12], the scope is enlarged to the affine case.

The choice of the best representation from multiple ones follows the principle "breefs" bracket-oriented representation, elimination and expansion for factored and shortest results. 
In this paper, this principle is explained in great details in the procedure of automated theorem proving in projective and affine conic geometries.

The main content of this paper is some further exploration of conic geometry from both projective and affine aspects, and the illustration of the "breefs" principle by detailed case studies. The focus is concentrated on how to carry out the principle to simply algebraic computations.

\section{Cayley and bracket algebras for projective and affine geometries}

We present a brief introduction of Cayley algebra and bracket algebra and their connections with projective geometry and affine geometry. Thorough expositions can be found in [7], [1], [17], [20], etc.

Let $\mathcal{V}^{n}$ be an $n$-dimensional vector space over a field $\mathcal{F}$ whose characteristic is not 2. Then $\mathcal{V}$ generates a Grassmann algebra $\Lambda\left(\mathcal{V}^{n}\right)$ in which the outer product is denoted by juxtaposition of elements. The Grassmann space is graded, whose grades range from 0 to $n$. An element of grade $r$ is called an $r$-vector.

Let $\langle x\rangle_{r}$ denote the $r$-graded part of $x \in \Lambda\left(\mathcal{V}^{n}\right)$. Let $I_{n}$ be a fixed nonzero $n$-vector in $\Lambda\left(\mathcal{V}^{n}\right)$. The following bilinear form

$$
B_{I_{n}}(x, y)=\langle x \vee y\rangle_{n} / I_{n}, \forall x, y \in \Lambda\left(\mathcal{V}^{n}\right)
$$

is nonsingular, and induces a linear invertible mapping $i: \Lambda\left(\mathcal{V}^{n}\right) \longrightarrow \Lambda\left(\mathcal{V}^{n *}\right)$, where $\mathcal{V}^{n *}$ is the dual vector space of $\mathcal{V}^{n}$. Then $\Lambda=i^{-1} \circ \vee \circ i$ defines the meet product in $\Lambda\left(\mathcal{V}^{n}\right)$. The Grassmann space $\Lambda\left(\mathcal{V}^{n}\right)$ equipped with the outer product and the meet product is called the Cayley algebra over $\mathcal{V}^{n}$.

Cayley algebra provides projectively invariant algebraic interpretations of synthetic geometric statements. In this algebra, a projective point is represented by a nonzero vector, which is unique up to scale. It is always denoted by a bold-faced integer or character. A line passing through points $\mathbf{1 , 2}$ is represented by $\mathbf{1 2}$. Three points $\mathbf{1 , 2 , 3}$ are collinear if and only if their outer product $\mathbf{1 2 3}$ equals zero.

In the projective plane, the intersection of two lines $\mathbf{1 2}, \mathbf{1}^{\prime} \mathbf{2}^{\prime}$ is $\mathbf{1 2} \wedge \mathbf{1}^{\prime} \mathbf{2}^{\prime}$. For three lines $12,1^{\prime} \mathbf{2}^{\prime}, \mathbf{1}^{\prime \prime} \mathbf{2}^{\prime \prime}$, their meet product $12 \wedge 1^{\prime} \mathbf{2}^{\prime} \wedge \mathbf{1}^{\prime \prime} \mathbf{2}^{\prime \prime}$ equals zero if and only if the three lines are concurrent.

An affine space $\mathcal{A}^{n}$ is composed of the projective points outside a projective hyperplanes in an $n \mathrm{D}$ projective space. In Cayley algebra, a projective hyperplane in the projective space $\mathcal{V}^{n+1}$ is represented by a nonzero $n$-vector $I_{n}$. Projective points in this hyperplane are called points at infinity of $\mathcal{A}^{n}$, and the hyperplane is called the hyperplane at infinity. So a vector $\mathbf{X}$ represents a point in $\mathcal{A}^{n}$ if and only if $\mathbf{X} I_{n}=0$. The point at infinity of line $\mathbf{1 2}$ is $I_{n} \wedge \mathbf{1 2}$. Two coplanar lines 12, 34 are parallel if they meet at infinity, i.e., if $(\mathbf{1 2} \wedge \mathbf{3 4}) I_{n}=0$.

Now we introduce bracket algebra, a suitable coordinate-free algebraic setting to deal with projective configurations. For any $n$-vector $J_{n} \in \Lambda\left(\mathcal{V}^{n}\right)$, its bracket is defined by

$$
\left[J_{n}\right]=J_{n} / I_{n}=B_{I_{n}}\left(J_{n}, 1\right)=B_{I_{n}}\left(1, J_{n}\right) .
$$

The following is the Cramer's rule: for any $n+1$ vectors $\mathbf{A}_{1}, \ldots, \mathbf{A}_{n}, \mathbf{B}$ in $\mathcal{V}^{n}$,

$$
\left[\mathbf{A}_{1} \cdots \mathbf{A}_{n}\right] \mathbf{B}=\sum_{i=1}^{n}(-1)^{i+1}\left[\mathbf{B} \mathbf{A}_{1} \cdots \check{\mathbf{A}}_{i} \cdots \mathbf{A}_{n}\right] \mathbf{A}_{i} .
$$


Here $\check{\mathbf{A}}_{i}$ denotes the missing of $\mathbf{A}_{i}$ in the series $\mathbf{A}_{1}$ to $\mathbf{A}_{n}$. The following is the expansion formula of the meet product: for vectors $\mathbf{A}_{1}, \ldots, \mathbf{A}_{r}$ and $\mathbf{B}_{1}, \ldots, \mathbf{B}_{s}$, where $r+s \geq n$,

$$
\begin{aligned}
& \left(\mathbf{A}_{1} \cdots \mathbf{A}_{r}\right) \wedge\left(\mathbf{B}_{1} \cdots \mathbf{B}_{s}\right) \\
= & \sum_{\sigma} \operatorname{sign}(\sigma)\left[\mathbf{A}_{\sigma(1)} \cdots \mathbf{A}_{\sigma(n-s)} \mathbf{B}_{1} \cdots \mathbf{B}_{s}\right] \mathbf{A}_{\sigma(n-s+1)} \cdots \mathbf{A}_{\sigma(r)} \\
= & \sum_{\tau} \operatorname{sign}(\tau)\left[\mathbf{A}_{1} \cdots \mathbf{A}_{r} \mathbf{B}_{\tau(r+s+1-n)} \cdots \mathbf{B}_{\tau(s)}\right] \mathbf{B}_{\tau(1)} \cdots \mathbf{B}_{\tau(r+s-n)} .
\end{aligned}
$$

Here $\sigma$ is a permutation of $1, \ldots, r$ such that $\sigma(1)<\cdots<\sigma(n-s)$ and $\sigma(n-s+1)<$ $\cdots<\sigma(r)$, and $\tau$ is a permutation of $1, \ldots, s$ such that $\tau(1)<\cdots<\tau(r+s-n)$ and $\tau(r+s+1-n)<\cdots<\tau(s)$.

Let $\mathbf{A}_{1}, \ldots, \mathbf{A}_{m}$ be symbols, and let $\left[\mathbf{A}_{i_{1}} \cdots \mathbf{A}_{i_{n}}\right]$ be indeterminates over $\mathcal{F}$ for each $n$-tuple $1 \leq i_{1}, \ldots, i_{n} \leq m$, such that they are algebraically independent over $\mathcal{F}$ and each $n$ tuple is anticommutative with respect to its elements. The $(n-1) D$ bracket algebra generated by the $\mathbf{A}$ 's over $\mathcal{F}$ is the quotient of the polynomial $\operatorname{ring} \mathcal{F}\left[\left\{\left[\mathbf{A}_{i_{1}} \cdots \mathbf{A}_{i_{n}}\right] \mid 1 \leq i_{1}, \ldots, i_{n} \leq\right.\right.$ $m\}$ ] by the ideal $\mathcal{I}_{n, m}$ generated by elements of the following three types:

B1. $\left[\mathbf{A}_{i_{1}} \cdots \mathbf{A}_{i_{n}}\right]$ if any $i_{j}=i_{k}, j \neq k$.

B2. $\left[\mathbf{A}_{i_{1}} \cdots \mathbf{A}_{i_{n}}\right]-\operatorname{sign}(\sigma)\left[\mathbf{A}_{i_{\sigma(1)}} \cdots \mathbf{A}_{i_{\sigma(n)}}\right]$ for any permutation $\sigma$ of $1, \ldots, n$.

GP. (Grassmann-Plücker polynomials)

$$
\sum_{k=1}^{n+1}(-1)^{k+1}\left[\mathbf{A}_{i_{1}} \cdots \mathbf{A}_{i_{n-1}} \mathbf{A}_{j_{k}}\right]\left[\mathbf{A}_{j_{1}} \ldots \check{\mathbf{A}}_{j_{k}} \ldots \mathbf{A}_{j_{n+1}}\right] .
$$

Let $I_{n}$ denote the hyperplane at infinity of $\mathcal{A}^{n}$ in $\mathcal{V}^{n+1}$. Define a linear mapping $\partial$ from $\Lambda\left(\mathcal{V}^{n+1}\right)$ to $\Lambda\left(I_{n}\right)$, called the boundary operator, as follows:

$$
\partial(A)=I_{n} \wedge A, \forall A \in \Lambda\left(\mathcal{V}^{n+1}\right) .
$$

When $A$ is a vector, we usually use the notation $\partial(\mathbf{A})=[\mathbf{A}]$, although the bracket here is no longer in the same sense as before. In application, people usually set $[\mathbf{A}]=1$ for all points in $\mathcal{A}^{n}$. Then the representation becomes inhomogeneous.

Bracket algebra can be extended to the affine case as follows. Let $\mathbf{A}_{1}, \ldots, \mathbf{A}_{m}$ be symbols, and let $\left[\mathbf{A}_{i_{1}} \cdots \mathbf{A}_{i_{n}}\right]$ be indeterminates over $\mathcal{F}$ for each $n$-tuple $1 \leq i_{1}, \ldots, i_{n} \leq m$, such that they are algebraically independent over $\mathcal{F}$ and each $n$-tuple is anticommutative with respect to its elements. The $(n-1) D$ affine bracket algebra generated by the $\mathbf{A}$ 's over $\mathcal{F}$ is the quotient of the polynomial ring $\mathcal{F}\left[\left\{\left[\mathbf{A}_{i_{1}} \cdots \mathbf{A}_{i_{n}}\right],\left[\mathbf{A}_{j}\right] \mid 1 \leq i_{1}, \ldots, i_{n} \leq m, 1 \leq j \leq m\right\}\right]$ by the ideal $\mathcal{J}_{n, m}$ generated by elements of four types: B1, B2, GP, and

AGP. (Affine Grassmann-Plücker polynomials)

$$
\sum_{k=1}^{n+1}(-1)^{k+1}\left[\mathbf{A}_{j_{k}}\right]\left[\mathbf{A}_{j_{1}} \ldots \check{\mathbf{A}}_{j_{k}} \ldots \mathbf{A}_{j_{n+1}}\right] .
$$




\section{Some algebraic representations of conics}

Algebraic representations of geometric entities or constraints are needed in algebraic computations of geometric problems. There are often multiple representations for the same geometric entity or constraint, and different representations can occur within the same expression. It is an important task to study various representations and the transformation properties among them.

\subsection{Point conics}

There are three kinds of projective conics: (1) a line and itself, (2) two different lines, (3) a conic having no lines, called nondegenerate conic. Only the latter two are considered in this paper. The numbers field can be allowed to be any field of characteristic not 2 .

Let $\mathbf{1}, \mathbf{2}, \mathbf{3}, \mathbf{4}, \mathbf{5}$ be five points in the projective plane. Assume that they are distinct from each other and that no four of them are collinear. This set of inequality conditions is denoted by $\exists \mathbf{1 2 3 4 5}$. A classical conclusion is that such five points determine a unique conic, denoted by conic(12345), such that any point $\mathbf{X}$ in the plane is on the conic if and only if

$$
\operatorname{conic}(\mathbf{1 2 3 4 5 X})=0 \text {. }
$$

Such a conic is called a point conic. For any six points $\mathbf{1 , 2}, \mathbf{3}, \mathbf{4}, \mathbf{5}, \mathbf{X}$ in the plane, the expression conic $(\mathbf{1 2 3 4 5} \mathbf{X})$ is antisymmetric with respect to them.

Two distinct points $\mathbf{A}, \mathbf{B}$ are said to be conjugate with respect to a conic, if either they are conjugate with respect to the points $\mathbf{C}, \mathbf{D}$ in which $\mathbf{A B}$ meets the conic, or line $\mathbf{A B}$ is part of the conic. A point is conjugate to itself with respect to a conic if it is on the conic.

If $\mathbf{A}$ is not a double point of a conic, i.e., not the intersection of the two lines of a conic, then the conjugates of $\mathbf{A}$ with respect to the conic form a line, called the polar of $\mathbf{A}$. In particular, if $\mathbf{A}$ is on the conic, its polar is the tangent at $\mathbf{A}$. Dually, the points on a line $l$ which is not part of a conic, have a unique common conjugate with respect to the conic, called the pole of $l$. When $l$ is tangent to the conic, its pole is the point of tangency.

By [11], the pole of line $\mathbf{1 2}$ with respect to conic(12345) can be represented as follows:

$$
\operatorname{pole}_{12,345}=[\mathbf{1 4 5}][234][235] 1+[134][135][245] 2-[124][125][345] 3 .
$$

The representation is symmetric with respect to $\mathbf{1}, \mathbf{2}$, antisymmetric with respect to $\mathbf{3 , 4}, \mathbf{5}$, and follows the point-conic transformation rules with respect to $\mathbf{3 , 4 , 5}$.

Point-conic transformation rules. Let $C(\mathcal{S})$ be a Cayley or bracket expression of points $\mathcal{S}=\mathbf{1}, \ldots, \mathbf{i}$ on a conic, and assume that $C(\mathcal{S})$ is either symmetric or antisymmetric with respect to $\mathcal{S}$. The point-conic transformation rules of $C(\mathcal{S})$ with respect to $\mathcal{S}$ are that for any conic points $\mathbf{1}^{\prime}, \mathbf{2}^{\prime}, \mathbf{3}^{\prime}$,

$$
\frac{C(\mathbf{1}, \mathbf{2}, \ldots, \mathbf{i})}{C\left(\mathbf{1}^{\prime}, \mathbf{2}, \ldots, \mathbf{i}\right)}=\frac{\left[\mathbf{1} \mathbf{k}_{1} \mathbf{k}_{2}\right]\left[\mathbf{1} \mathbf{k}_{3} \mathbf{k}_{4}\right]}{\left[\mathbf{1}^{\prime} \mathbf{k}_{1} \mathbf{k}_{2}\right]\left[\mathbf{1}^{\prime} \mathbf{k}_{3} \mathbf{k}_{4}\right]}
$$

where $\mathbf{k}_{1}, \ldots, \mathbf{k}_{4}$ are any four elements in $\mathcal{S}$ different from $\mathbf{1}, \mathbf{1}^{\prime}$; and

$$
\frac{C(\mathbf{1}, \mathbf{2}, \mathbf{3}, \ldots, \mathbf{i})}{C\left(\mathbf{1}^{\prime}, \mathbf{2}^{\prime}, \mathbf{3}, \ldots, \mathbf{i}\right)}=\frac{\left[\mathbf{1} \mathbf{k}_{3}\right]\left[1 \mathbf{k}_{1} \mathbf{k}_{2}\right]\left[\mathbf{2} \mathbf{k}_{1} \mathbf{k}_{2}\right]}{\left[\mathbf{1}^{\prime} \mathbf{2}^{\prime} \mathbf{k}_{3}\right]\left[\mathbf{1}^{\prime} \mathbf{k}_{1} \mathbf{k}_{2}\right]\left[\mathbf{2}^{\prime} \mathbf{k}_{1} \mathbf{k}_{2}\right]},
$$


where $\mathbf{k}_{1}, \mathbf{k}_{2}, \mathbf{k}_{3}$ are any three elements in $\mathcal{S}$ different from $\mathbf{1}, \mathbf{2}, \mathbf{1}^{\prime}, \mathbf{2}^{\prime} ;$ and

$$
\frac{C(\mathbf{1}, \mathbf{2}, \mathbf{3}, \mathbf{4}, \ldots, \mathbf{i})}{C\left(\mathbf{1}^{\prime}, \mathbf{2}^{\prime}, \mathbf{3}^{\prime}, \mathbf{4}, \ldots, \mathbf{i}\right)}=\frac{[\mathbf{1 2 3}]\left[\mathbf{1} \mathbf{k}_{1} \mathbf{k}_{2}\right]\left[\mathbf{2} \mathbf{k}_{1} \mathbf{k}_{2}\right]\left[3 \mathbf{k}_{1} \mathbf{k}_{2}\right]}{\left[\mathbf{1}^{\prime} \mathbf{2}^{\prime} \mathbf{3}^{\prime}\right]\left[\mathbf{1}^{\prime} \mathbf{k}_{1} \mathbf{k}_{2}\right]\left[\mathbf{2}^{\prime} \mathbf{k}_{1} \mathbf{k}_{2}\right]\left[\mathbf{3}^{\prime} \mathbf{k}_{1} \mathbf{k}_{2}\right]}
$$

where $\mathbf{k}_{1}, \mathbf{k}_{2}$ are any two elements in $\mathcal{S}$ different from $\mathbf{1}, \mathbf{2}, \mathbf{3}, \mathbf{1}^{\prime}, \mathbf{2}^{\prime}, \mathbf{3}^{\prime}$. The ratios in the formulas are called transformation coefficients. In the three cases, the nondegeneracy requirements are respectively

$$
\begin{array}{ll}
\exists \mathbf{1} \mathbf{k}_{1} \mathbf{k}_{2} \mathbf{k}_{3} \mathbf{k}_{4}, & \exists \mathbf{1}^{\prime} \mathbf{k}_{1} \mathbf{k}_{2} \mathbf{k}_{3} \mathbf{k}_{4} \\
\exists \mathbf{1} \mathbf{2} \mathbf{k}_{1} \mathbf{k}_{2} \mathbf{k}_{3}, & \exists \mathbf{1}^{\prime} \mathbf{2}^{\prime} \mathbf{k}_{1} \mathbf{k}_{2} \mathbf{k}_{3} \\
\exists \mathbf{1 2} \mathbf{2} \mathbf{k}_{1} \mathbf{k}_{2}, & \exists \mathbf{1}^{\prime} \mathbf{2}^{\prime} \mathbf{3}^{\prime} \mathbf{k}_{1} \mathbf{k}_{2} .
\end{array}
$$

Let $\mathbf{X}$ be the second intersection of line $\mathbf{A B}$ with conic(A1234). In [11], the following representation of $\mathbf{X}$ is derived:

$$
\mathrm{X}_{\mathrm{AB}, 1234}=[134][24 \mathrm{~A}][3 \mathrm{AB}] 12 \wedge \mathrm{AB}-[124][34 \mathrm{~A}][2 \mathrm{AB}] 13 \wedge \mathrm{AB} .
$$

The representation is antisymmetric with respect to $\mathbf{1}, \mathbf{2}, \mathbf{3}, \mathbf{4}$ and satisfies the point-conic transformation rules with respect to the four points.

\subsection{Point-tangent conics}

A conic can also be constructed by four points and one tangent through one of the points. In [11], it is proved that given five points $\mathbf{1}, \mathbf{2}, \mathbf{3}, \mathbf{4 , 5}$ in the projective plane, such that (1) $\mathbf{1}, \mathbf{2}, \mathbf{3}, \mathbf{4}$ are distinct and non-collinear, (2) $\mathbf{4 , 5}$ are distinct, (3) either points $\mathbf{1}, \mathbf{2}, \mathbf{3}$ are not on line 45 , and point 4 is not on any of the lines $\mathbf{1 2}, \mathbf{1 3}, \mathbf{2 3}$, or only one of the points $\mathbf{1 , 2 , 3}$ is on line $\mathbf{4 5}$, then there exists a unique conic passing through points $\mathbf{1}, \mathbf{2}, \mathbf{3}, \mathbf{4}$ and tangent to line 45. A conic constructed in this manner is called a point-tangent conic, and denoted by conic $(\mathbf{1 2 3 4}, \mathbf{4 5})$. A point $\mathbf{X}$ is on the conic if and only if

$$
\operatorname{conic}(\mathrm{X} 1234,45)=[134][\mathbf{2 4 5}][\mathbf{1 4 X}][23 \mathrm{X}]-[\mathbf{2 3 4}][\mathbf{1 4 5}][\mathbf{1 3 X}][\mathbf{2 4 X}]=0 .
$$

The expression conic $(\mathbf{X 1 2 3 4}, \mathbf{4 5})$ is antisymmetric with respect to $\mathbf{1}, \mathbf{2}, \mathbf{3}, \mathbf{X}$.

For point-tangent conics, the transformation rules and the representations of various geometric entities can be similarly developed. As an example, we establish the representations of the second intersection of a line and a conic.

Let $\mathbf{X}=\operatorname{conic}(\mathbf{A 2 3 4}, \mathbf{4 5}) \cap \mathbf{A B}$. Then

$$
\operatorname{conic}(\mathrm{AX234}, \mathbf{4 5})=[34 \mathrm{X}][245][4 \mathrm{AX}][23 \mathrm{~A}]-[234][45 \mathrm{X}][3 \mathrm{AX}][24 \mathrm{~A}]=0
$$

Substituting $\mathbf{X}=\lambda \mathbf{A}+\mu \mathbf{B}$ into it, we get

$$
\frac{\lambda}{\mu}=\frac{[234][24 \mathrm{~B}][3 \mathrm{AB}][45 \mathrm{~A}]-[23 \mathrm{~B}][245][34 \mathrm{~A}][4 \mathrm{AB}]}{[23 \mathrm{~A}][245][34 \mathrm{~A}][4 \mathrm{AB}]-[234][24 \mathrm{~A}][3 \mathrm{AB}][45 \mathrm{~A}]}
$$

So

$$
\mathrm{X}=\mathrm{X}_{\mathrm{AB}, 2435}=[234][3 \mathrm{AB}][45 \mathrm{~A}] 24 \wedge \mathrm{AB}-[245][34 \mathrm{~A}][4 \mathrm{AB}] 23 \wedge \mathrm{AB}
$$


Similarly, from conic $(\mathbf{A X 2 3 4}, \mathbf{4 5})=\operatorname{conic}(\mathbf{A 3 2 X} 4, \mathbf{4 5})=0$, we get

$$
\mathrm{X}_{\mathrm{AB}, 4352}=[234][24 \mathrm{~A}][3 \mathrm{AB}] 45 \wedge \mathrm{AB}-[23 \mathrm{~A}][245][4 \mathrm{AB}] 34 \wedge \mathrm{AB},
$$

and

$$
\mathrm{X}_{\mathrm{AB}, 4235}=[24 \mathrm{~A}][345][3 \mathrm{AB}] 24 \wedge \mathrm{AB}-[245][2 \mathrm{AB}][34 \mathrm{~A}] 34 \wedge \mathrm{AB} .
$$

In the above indexing, $\mathbf{4}$ always precedes but does not immediately precede $\mathbf{5}$. There are all together 6 such indices. The relations are

$$
\mathrm{X}_{\mathrm{AB}, 4235}=-\mathrm{X}_{\mathrm{AB}, 4325}=-\mathrm{X}_{\mathrm{AB}, 2435}=\mathrm{X}_{\mathrm{AB}, 3425}=-\mathrm{X}_{\mathrm{AB}, 4253}=\mathrm{X}_{\mathrm{AB}, 4352} \text {, }
$$

i.e., $\mathbf{X}_{\mathrm{AB}, \mathbf{4 2 3 5}}$ is antisymmetric with respect to $\mathbf{4 , 2 , 3 , 5}$.

\subsection{Tangent-point conics}

The third construction of a conic is by two tangents and a point. In [11], it is proved that given five points $\mathbf{1}, \mathbf{2}, \mathbf{3}, \mathbf{4}, \mathbf{5}$ in the projective plane, such that (1) $\mathbf{1}, \mathbf{2}, \mathbf{3}$ are not collinear, (2) 24, 35 are lines and are distinct, (3) either $\mathbf{1}$ is on one of the lines $\mathbf{2 4 , 3 5}$, or $\mathbf{1}, \mathbf{3}$

are not on line $\mathbf{2 4}$ and $\mathbf{1 , 2}$ are not on line 35, then there exists a unique conic passing through 1, 2, 3 and tangent to lines $\mathbf{2 4 , 3 5}$. A conic constructed in this manner is called a tangent-point conic, denoted by conic $(\mathbf{1 2 3}, \mathbf{2 4}, \mathbf{3 5})$. A point $\mathbf{X}$ is on the conic if and only if $\operatorname{conic}(\mathbf{X} 123, \mathbf{2 4}, \mathbf{3 5})=0$, where

$$
\begin{aligned}
& \operatorname{conic}(\mathbf{X 1 2 3}, \mathbf{2 4}, \mathbf{3 5}) \\
= & {[\mathbf{1 2 3}]^{2}[\mathbf{2 4 X}][\mathbf{3 5 X}]-[\mathbf{1 2 4}][\mathbf{1 3 5}][\mathbf{2 3 X}]^{2} } \\
= & {[\mathbf{1 2 X}][\mathbf{1 3 X}][\mathbf{2 3 4}][\mathbf{2 3 5}]-[\mathbf{1 2 X}][\mathbf{2 3 X}][\mathbf{1 3 5}][\mathbf{2 3 4}]-[\mathbf{1 3 X}][\mathbf{2 3 X}][\mathbf{1 2 4}][\mathbf{2 3 5}] }
\end{aligned}
$$

The expression conic $(\mathbf{X} \mathbf{1 2 3}, \mathbf{2 4}, \mathbf{3 5})$ is symmetric with respect to $\mathbf{2 4}$ and $\mathbf{3 5}$.

Again we compute the second intersection of a line and a conic. Let $\mathbf{X}=\mathbf{A B} \cap$ conic $(\mathbf{A 2 3}, \mathbf{2 4}, \mathbf{3 5})$. Using the second expression of conic $(\mathbf{X A 2 3 , 2 4 , 3 5 )}$ in $(24)$, we get

$$
\mathrm{X}_{\mathrm{AB}, 24,35}=[234][2 \mathrm{AB}][35 \mathrm{~A}] \mathrm{AB} \wedge 23+[235][23 \mathrm{~A}][3 \mathrm{AB}] \mathrm{AB} \wedge 24 .
$$

The expression is symmetric with respect to $\mathbf{2 4}$ and $\mathbf{3 5}$.

\subsection{Affine conics}

In affine geometry, there are three kinds of nondegenerate conics: (1) ellipse, if the line at infinity does not meet the conic; 2 ) hyperbola, if the line at infinity meets the conic at two distinct points at infinity; (3) parabola, if the line at infinity is tangent to the conic.

The pole of the line at infinity with respect to a conic is called the center of the conic. The center of an ellipse or hyperbola is a point, while the center of a parabola is a point at infinity. An affine line passing through the center is called a diameter of the conic. Two diameters are conjugate to each other if their points at infinity are conjugate to each other. For a hyperbola, the two tangents at its points at infinity are called the asymptotes.

Similar to the projective case, various representations and their transformation properties can be established. As an example, let us find the representations of a parabola by its center $\mathbf{0}$ and three points $\mathbf{1}, \mathbf{2}, \mathbf{3}$. Let $\mathbf{4}$ be a point at infinity different from $\mathbf{0}$. Then the parabola is just conic $(\mathbf{1 2 3 0}, \mathbf{0 4})$. 
Let $\mathbf{1}, \mathbf{2}, \mathbf{3}$ be points in $\mathcal{A}^{2}$, and let $\mathbf{0}$ be a point at infinity, such that no three of $\mathbf{1 , 2}, \mathbf{3}, \mathbf{0}$ are collinear. Then there exists a unique parabola passing through $\mathbf{1}, \mathbf{2}, \mathbf{3}$ with $\mathbf{0}$ as the center. Any point $\mathbf{X}$ is on the parabola if and only if

$$
\operatorname{parabola}(\mathbf{X} \mathbf{1 2 3}, \mathbf{0})=[\mathbf{2}][\mathbf{1 3 0}][\mathbf{1 0 X}][\mathbf{2 3 X}]-[\mathbf{1}][\mathbf{2 3 0}][\mathbf{2 0 X}][\mathbf{1 3 X}]=0 .
$$

The expression parabola $(\mathbf{X 1 2 3 , 0})$ is antisymmetric with respect to $\mathbf{1}, \mathbf{2}, \mathbf{3}, \mathbf{X}$.

\section{Case study 1}

Example 1. Let there be a hyperbola whose two asymptotes are $l_{1}, l_{2}$ and whose center is $\mathbf{0}$. Let $\mathbf{1}, \mathbf{2}, \mathbf{3}$ be any three points on the hyperbola. Let $\mathbf{6}=\mathbf{1 2} \cap l_{1}$, and let $\mathbf{7}$ be the intersection of the parallel line of $l_{1}$ through $\mathbf{3}$ and the parallel line of $l_{2}$ through $\mathbf{1}$. Then $\mathbf{2 3}$ and $\mathbf{6 7}$ are parallel.

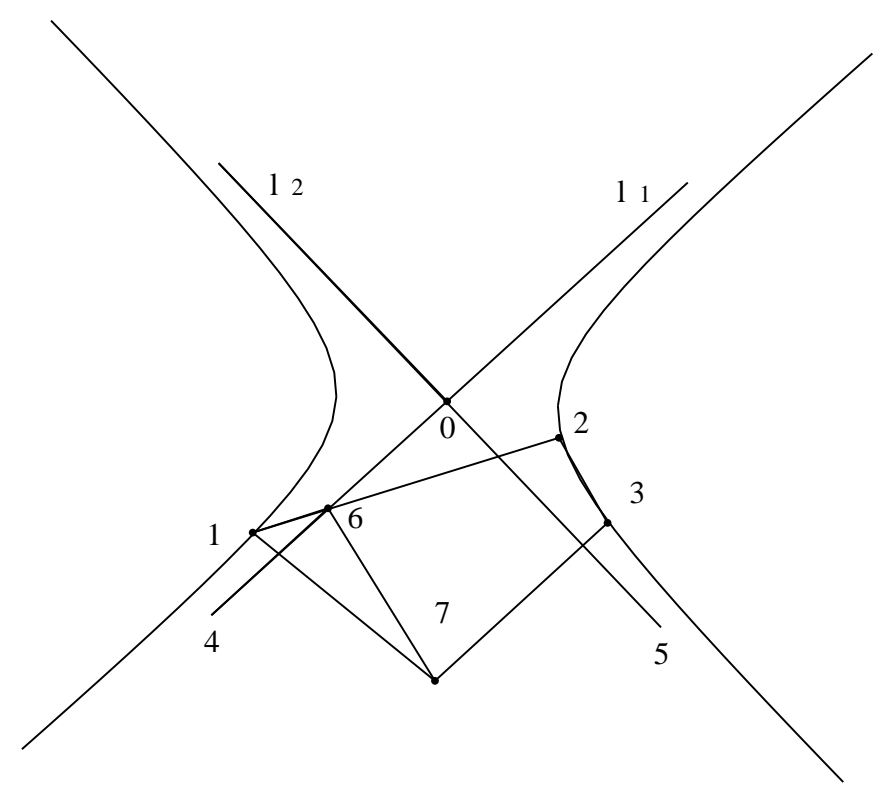

Fig. 1. Example 1.

Hypotheses:

Free points: $\mathbf{1}, \mathbf{2}, \mathbf{3}$.

Free points at infinity: $\mathbf{4 , 5}$.

Pole: $\mathbf{0}=$ pole $_{\mathbf{4 5}}(\mathbf{1 2 3 4 5})$.

Intersections: $\mathbf{6}=\mathbf{1 2} \cap 04, \quad 7=34 \cap 15$.

Conclusion: $[\mathbf{2 3} \wedge \mathbf{6 7}]=0$.

Proof: 


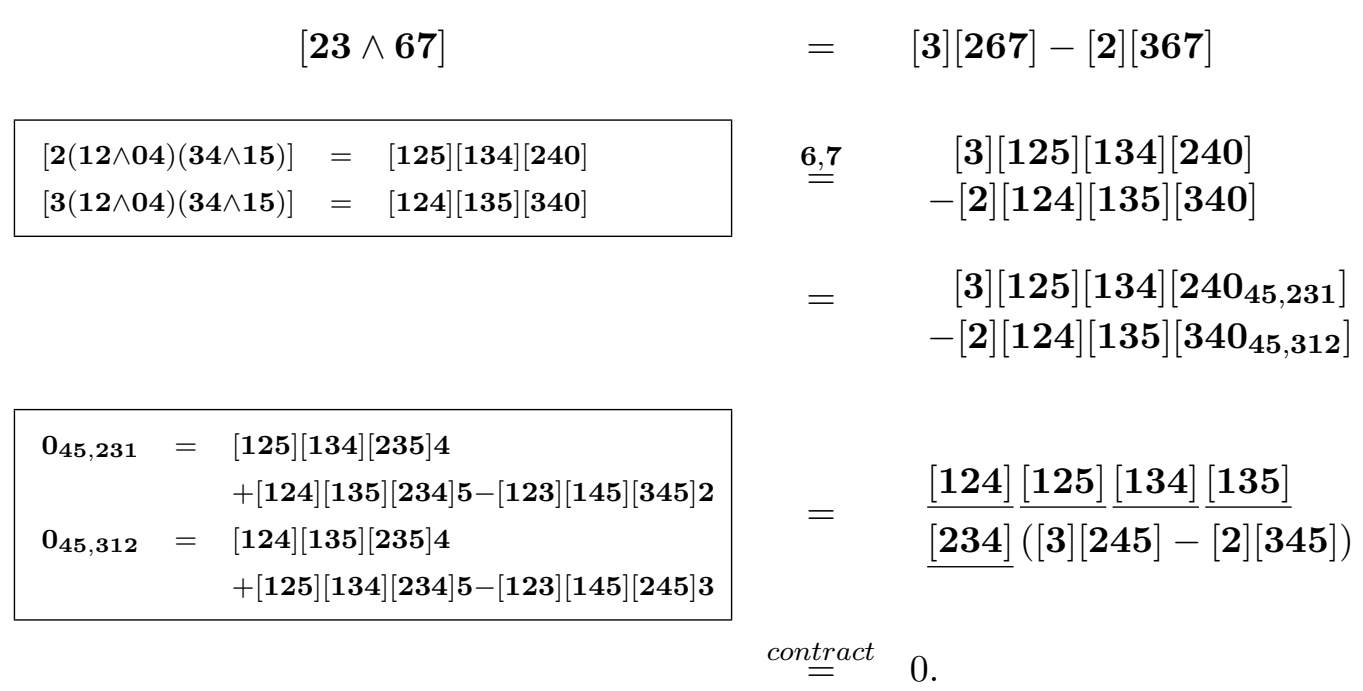

Additional nondegeneracy condition: none.

Explanation and discussion:

(1) $23 \wedge 67$ has two different expansions. In the above proof, the expansion separating $\mathbf{2}, \mathbf{3}$ is used.

(2) The second step is eliminating $\mathbf{6}, \mathbf{7}$ at the same time. The reason for this batch elimination is that $\mathbf{6}, \mathbf{7}$ are both at the end of the sequence of constructions:

$$
1,2,3,4,5 \prec\left\{\begin{array}{l}
0 \prec 6 ; \\
7 ;
\end{array}\right.
$$

and both are intersections of lines.

(3) Both [267] and [367] are of the type $\left[\mathbf{1}\left(\mathbf{1}^{\prime} \mathbf{2}^{\prime} \wedge \mathbf{3}^{\prime} \mathbf{4}^{\prime}\right)\left(\mathbf{1}^{\prime \prime} \mathbf{2}^{\prime \prime} \wedge \mathbf{3}^{\prime \prime} \mathbf{4}^{\prime \prime}\right)\right]$ in which one of the $\mathbf{i}^{\prime} \mathbf{s}$ or $\mathbf{i}^{\prime \prime}$ s equals $\mathbf{1}$. This is the recursion pattern [10] and always has monomial expansion.

(4) The third step is choosing representations for $\mathbf{0}$ before its elimination. This is carried out within each bracket containing $\mathbf{0}$.

By (12), $\mathbf{0}$ is a linear combination of $\mathbf{4 , 5}$ and another conic point $\mathbf{X}$, so it has two essential points $\mathbf{4}, \mathbf{5}$, which occur in every representation of $\mathbf{0}$.

In $[\mathbf{2 4 0}]$, because of the occurrence of conic point $\mathbf{2}$, it is chosen as $\mathbf{X}$. In [340], $\mathbf{3}$ is chosen as $\mathbf{X}$. The result is that both brackets have monomial expansions after the elimination of $\mathbf{0}$.

(5) The last step is a contraction. It is based on the following Grassmann-Plücker syzygy

$$
[3][245]-[2][345]=[4][235]-[5][234],
$$

and the bracket computation rules $[\mathbf{4}]=[\mathbf{5}]=0$. The purpose is to reduce the number of terms of a polynomial within bracket algebra.

(6) In every step, if there is any common bracket factor, it is automatically removed. In the above proof, all such factors are outlined. 
(7) Additional nondegeneracy conditions: These are some inequality requirements which are needed not by the geometric constructions, but by the algebraic proof. In this example, there is no additional nondegeneracy condition.

An alternative proof is to expand $23 \wedge \mathbf{6 7}$ by separating 6,7 :

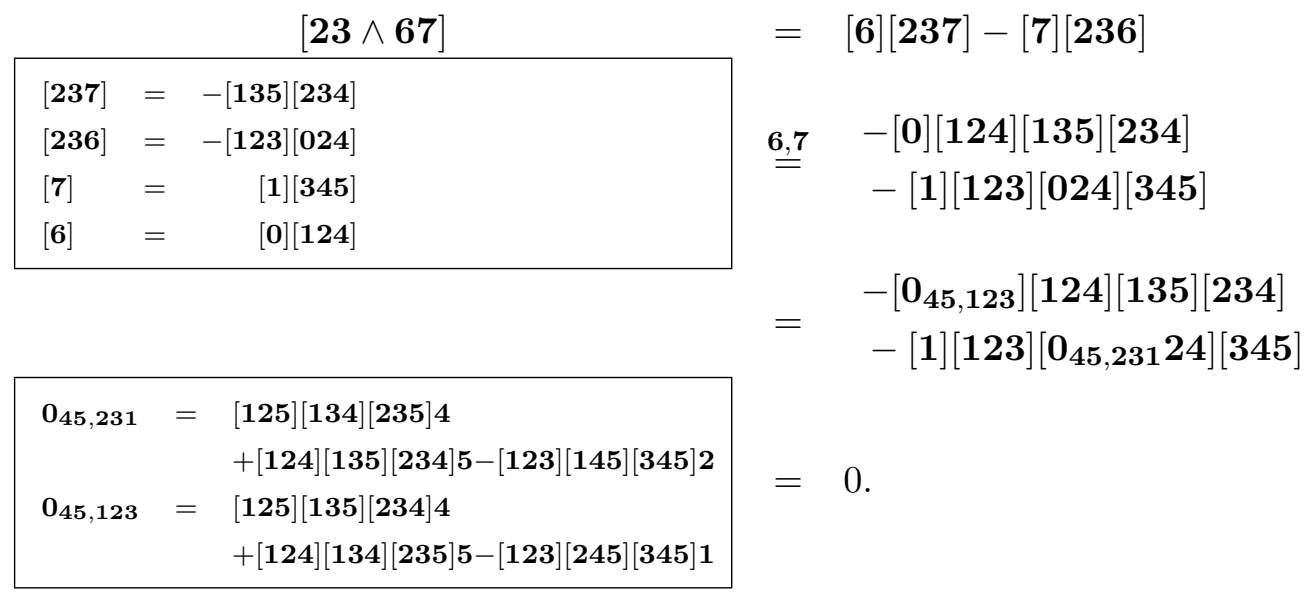

Further discussion:

(8) The second proof is simpler. By experience, if there are only two ends, then the expansion which separates them often performs better. In the above, all brackets containing 6, 7 have monomial expansions.

(9) There are two brackets containing 0: $[\mathbf{0}]$ and $[\mathbf{0 2 4}]$. The latter bracket demands the representation of $\mathbf{0}$ by $\mathbf{4 , 5 , 2}$. The former bracket does not demand anything, because the conic has only two points at infinity $\mathbf{4 , 5}$, and they are both essential points of $\mathbf{0}$. However, choosing the representation by $\mathbf{4 , 5}, \mathbf{1}$ can avoid doing contraction later on.

(10) The projective version of the theorem is to change $\mathbf{4 , 5}$ to two free points in the projective plane, and change the conclusion to the concurrency of lines $\mathbf{2 3 , 4 5 , 6 7}$. The proof is the same.

\section{Case study 2}

Example 2. Let there be a parabola passing through points $\mathbf{2}, \mathbf{3}, \mathbf{4}$. Let $\mathbf{7}$ be the intersection of the tangents at $\mathbf{3}, \mathbf{4}$, and let $\mathbf{8}$ be the intersection of $\mathbf{2 3}$ with the diameter through $\mathbf{4}$. Then $\mathbf{7 8}$ is parallel to the tangent at $\mathbf{2}$.

Hypotheses:

Free points: $\mathbf{3}, \mathbf{4}, \mathbf{5}$.

Free points at infinity: $\mathbf{1}, \mathbf{6}$.

Tangent point: $\mathbf{2}$ is the second tangent point from 6 to conic $(\mathbf{3 4 5 1}, \mathbf{1 6})$.

Pole: $7=$ pole $_{34}(3451,16)$.

Intersection: $\mathbf{8}=\mathbf{1 4} \cap \mathbf{2 3}$.

Conclusion: $[678]=0$.

Proof. 




Fig. 2. Example 2.

$[678]$

$$
\begin{aligned}
\stackrel{8}{=} & 67 \wedge 14 \wedge 23 \\
= & {[146][237]-[147][236] } \\
= & \quad\left[146_{12,435}\right]\left[237_{34,215}\right] \\
& -\left[147_{34,125}\right]\left[236_{12,345}\right]
\end{aligned}
$$

$$
\begin{aligned}
& 6_{12,435}=-[135][234][245] 1 \\
& -[134][145][235] 2+[123][125][345] 4 \\
& 6_{12,345}=[145][234][235] 1 \\
& +[\mathbf{1 3 4}][\mathbf{1 3 5}][\mathbf{2 4 5}] 2-[\mathbf{1 2 4}][\mathbf{1 2 5}][\mathbf{3 4 5}] 3 \stackrel{6,7}{=} 0 . \\
& 7_{34,215}=-[124][135][245] 3 \\
& -[123][145][235] 4+[125][134][345] 2 \\
& 7_{34,125}=[124][145][235] 3 \\
& +[123][135][245] 4-[125][234][345] 1
\end{aligned}
$$

Additional nondegeneracy condition: $\exists \mathbf{1 2 3 4 5}$.

Explanation and discussion:

(1) The sequence of constructions is

$$
1,6,3,4,5 \prec\left\{\begin{array}{l}
2 \prec 8 ; \\
7 .
\end{array}\right.
$$

Because $\mathbf{7}$ has multiple representations, only end $\mathbf{8}$ is eliminated in the first step.

(2) $\mathbf{6}$ is pole $_{\mathbf{1 2}}(\mathbf{1 2 3 4 5})$, so the order of elimination is $\mathbf{1}, \mathbf{2}, \mathbf{3}, \mathbf{4}, \mathbf{5} \prec \mathbf{6}, \mathbf{7}$. The second step expands $\mathbf{6 7} \wedge \mathbf{1 4} \wedge \mathbf{2 3}$ by separating $\mathbf{6 , 7}$.

(3) The third step is choosing representations for $\mathbf{6}, \mathbf{7}$. In each bracket, the representation is uniquely determined.

(4) The fourth step is a batch elimination of $\mathbf{6}, \mathbf{7}$.

(5) In this proof, the order of elimination does not follow the order of construction. We can certainly represent $\mathbf{2 , 7}$ by $\mathbf{6}$ etc. Then the proof becomes slightly more complicated. 
In doing elimination, we can either follow the original construction order, or reset the order in favor of the conclusion. In programming, when the input constructions are given, the conics, tangents, poles, etc., are collected before any computation occurs. A conic is composed of all the points and tangents of it. In this example, the parabola has four points, one point at infinity, three tangents and one tangent at infinity. There are two poles: $\mathbf{6}$ and 7. The change of elimination order can then be made automatic by these information and the conclusion.

(6) In the proof, the property that $\mathbf{1 , 6}$ are points at infinity is nowhere used. So the projective version of the theorem is also proved.

(7) The requirement $\exists \mathbf{1 2 3 4 5}$ is not needed by the constructions in the hypotheses, but needed by the representations of $\mathbf{6}$ and $\mathbf{7}$ by the five conic points in the proof. It is an additional nondegeneracy condition.

\section{Case study 3}

Example 3. [Brianchon's Theorem] Let there be six points on a conic: $\mathbf{1 , 2 , 3 , 4 , 5 , 6}$. Draw six tangents of the conic at the six points. The neighboring tangents meet at points $\mathbf{7}, \mathbf{8}, \mathbf{9}, \mathbf{0}, \mathbf{A}, \mathbf{B}$ respectively. Then $\mathbf{9 B}, \mathbf{8 A}, \mathbf{7 0}$ are concurrent.

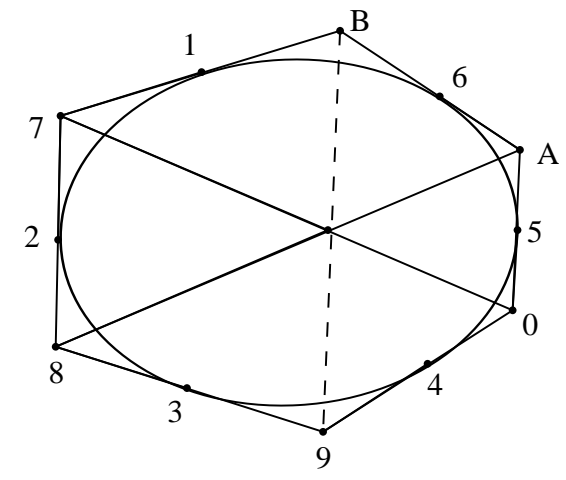

Fig. 3. Example 3.

Hypotheses:

Free conic points: $\mathbf{1 , 2}, \mathbf{3}, \mathbf{4}, \mathbf{5}, \mathbf{6}$.

Poles:

$$
\begin{array}{lll}
\mathbf{7}=\text { pole }_{12}(\mathbf{1 2 3 4 5 6}), & \mathbf{8}=\text { pole }_{\mathbf{2 3}}(\mathbf{1 2 3 4 5 6}), & \mathbf{9}=\operatorname{pole}_{\mathbf{3 4}}(\mathbf{1 2 3 4 5 6}) \\
\mathbf{0}=\operatorname{pole}_{45}(\mathbf{1 2 3 4 5 6}), & \mathbf{A}=\operatorname{pole}_{\mathbf{5 6}}(\mathbf{1 2 3 4 5 6}), \quad \mathbf{B}=\operatorname{pole}_{\mathbf{6 1}}(\mathbf{1 2 3 4 5 6})
\end{array}
$$

Conclusion: $\mathbf{9 B} \wedge \mathbf{8 A} \wedge \mathbf{7 0}=0$.

Proof. 


$$
\begin{aligned}
& 9 \mathrm{~B} \wedge 8 \mathrm{~A} \wedge 70 \\
& \stackrel{\text { expand }}{=} \quad[\mathbf{7 8 0}][\mathbf{9 A B}]-[\mathbf{7 0 A}][\mathbf{8 9 B}] \\
& =\quad\left[\begin{array}{lll}
7_{21,345} & 8_{23,145} & 0_{45,213}
\end{array}\right]\left[\begin{array}{lll}
9_{34,651} & A_{65,134} & B_{61,534}
\end{array}\right] \\
& -\left[\begin{array}{lll}
7_{12,546} & 0_{54,612} & A_{56,412}
\end{array}\right]\left[\begin{array}{lll}
8_{32,461} & 9_{34,261} & B_{61,324}
\end{array}\right] \\
& \times \frac{7_{21,345} 8_{23,145} 9_{34,651} 0_{45,213} A_{65,134} B_{61,534}}{7_{12,546} 8_{32,461} 9_{34,261} 0_{54,612} A_{56,412}, B_{61,324}} \\
& \underset{\mathbf{7}, \mathbf{8 , 9 , 0}, \mathrm{A}, \mathrm{B}}{=} 16 \underline{\left([\mathbf{1 2 4}][\mathbf{1 2 5}][\mathbf{1 3 4}]^{2}[\mathbf{1 3 6}][\mathbf{1 4 5}]^{2}[\mathbf{1 4 6}][\mathbf{2 3 5}][\mathbf{2 4 5}][\mathbf{3 4 6}]\right.} \\
& \times \underline{\left.[\mathbf{3 5 6}] /[\mathbf{1 2 6}]^{4}[\mathbf{2 3 4}]^{2}[\mathbf{4 5 6}]^{2}\right)} \\
& \times\left\{[\mathbf{1 2 6}]^{4}[\mathbf{1 3 5}]^{4}[\mathbf{2 3 4}]^{3}[\mathbf{2 4 5}][\mathbf{3 4 6}][\mathbf{4 5 6}]^{3}\right. \\
& \left.-[\mathbf{1 2 3}]^{3}[\mathbf{1 2 5}][\mathbf{1 3 6}][\mathbf{1 5 6}]^{3}[\mathbf{2 4 6}]^{4}[\mathbf{3 4 5}]^{4}\right\} \\
& \stackrel{\text { conic }}{=} \quad \underline{\mathbf{1 2 3}}]^{3}[\mathbf{1 5 6}]^{3}[\mathbf{2 4 6}]^{3}[\mathbf{3 4 5}]^{3}\{[\mathbf{1 2 6}][\mathbf{1 3 5}][\mathbf{2 4 5}][\mathbf{3 4 6}] \\
& -[125][136][246][345]\} \\
& \stackrel{\text { conic }}{=} \quad 0 .
\end{aligned}
$$

Additional nondegeneracy conditions: $\exists \mathbf{1 2 3 4 5}, \exists \mathbf{1 2 3 4 6}, \exists \mathbf{1 2 4 5 6}, \exists \mathbf{1 3 4 5 6}, \exists \mathbf{1 2 6}, \exists \mathbf{2 3 4}$, $\exists$ 456. Here $\exists \mathbf{1 2 6}$ denotes that $\mathbf{1 , 2}, \mathbf{6}$ are not collinear.

Explanation and discussion:

(1) $\mathbf{9 B} \wedge \mathbf{8 A} \wedge \mathbf{7 0}$ has three different expansions. Any expansion leads to much the same proof.

(2) The second step is choosing representations for all the points in the brackets before the batch elimination. In [780], the three points have essential points $\mathbf{1}^{1}, \mathbf{2}^{2}, \mathbf{3}^{1}, \mathbf{4}^{1}, \mathbf{5}^{1}$, where the exponents denote the numbers of occurrences in the bracket. So any of the three points should use the five conic points in the representation, and for $\mathbf{0}$, since $\mathbf{2}$ occurs twice in [780], it should be used definitely, i.e.,

$$
\mathbf{0}=\mathbf{0}_{45,213} .
$$

For $\mathbf{7}$ and $\mathbf{8}$, the representation cannot be uniquely determined. In the above proof, the following are used:

$$
7=7_{21,345}, 8=8_{23,145} .
$$

Same techniques apply to the other three brackets.

(3) It is a big burden to compute all the 4 brackets in the third line of the proof. The 
following are two formulas established in [11], where all the points are on the same conic.



$$
\begin{aligned}
& =-4\left[\mathbf{1}^{\prime \prime} \mathbf{2 4}\right][\mathbf{1 2 5}][134]\left[\mathbf{1}^{\prime \prime} 35\right]\left[\mathbf{1 4}^{\prime} 5^{\prime}\right]\left[14^{\prime \prime} 5^{\prime \prime}\right]\left[234^{\prime}\right]\left[234^{\prime \prime}\right]\left[235^{\prime}\right]\left[235^{\prime \prime}\right] \text {, } \\
& {\left[\text { pole }_{12,34^{\prime \prime} 5^{\prime \prime}} \text { pole }_{13,245^{\prime}} \text { pole }_{45,12^{\prime \prime} 3^{\prime \prime}}\right]} \\
& =-4[124]\left[12^{\prime \prime} 5\right][134]\left[13^{\prime \prime} 5\right]\left[14^{\prime \prime} 5^{\prime \prime}\right]\left[145^{\prime}\right]\left[2^{\prime \prime} 3^{\prime \prime} 4\right]\left[234^{\prime \prime}\right]\left[235^{\prime \prime}\right]\left[235^{\prime}\right] \text {. }
\end{aligned}
$$

By the above formulas, we have

$$
\begin{aligned}
& {\left[\begin{array}{lll}
\mathbf{7}_{\mathbf{2 1}, \mathbf{3 4 5}} & \mathbf{8}_{\mathbf{2 3 , 1 4 5}} & \mathbf{0}_{\mathbf{4 5 , 2 1 3}}
\end{array}\right]=-4[\mathbf{1 2 4}][\mathbf{1 2 5}][\mathbf{1 3 4}]^{2}[\mathbf{1 3 5}]^{2}[\mathbf{2 3 4}][\mathbf{2 3 5}][\mathbf{2 4 5}]^{2}}
\end{aligned}
$$

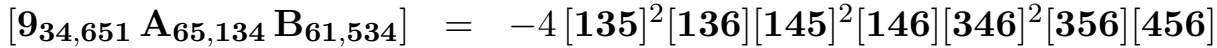

$$
\begin{aligned}
& {\left[\begin{array}{lll}
\mathbf{7}_{\mathbf{1 2}, \mathbf{5 4 6}} & \mathbf{0}_{\mathbf{5 4 , 6 1 2}} & \mathbf{A}_{\mathbf{5 6}, 412}
\end{array}\right]=-4[\mathbf{1 2 5}]^{2}[\mathbf{1 4 5}][\mathbf{1 4 6}]^{2}[\mathbf{1 5 6}][\mathbf{2 4 5}][\mathbf{2 4 6}]^{2}[\mathbf{2 5 6}]} \\
& {\left[\begin{array}{lll}
\mathbf{8}_{\mathbf{3 2}, 461} & \mathbf{9}_{\mathbf{3 4 , 2 6 1}} & \mathrm{B}_{61,324}
\end{array}\right]=-4[\mathbf{1 2 3}][\mathbf{1 2 4}]^{2}[\mathbf{1 3 4}][\mathbf{1 3 6}]^{2}[\mathbf{2 3 6}][\mathbf{2 4 6}]^{2}[\mathbf{3 4 6}]}
\end{aligned}
$$

(4) In the third line, all the six points have changed their representations, so the second term is multiplied by the transformation coefficients

$$
\begin{aligned}
k & =\frac{\mathbf{7}_{\mathbf{2 1 , 3 4 5}} \mathbf{8}_{\mathbf{2 3 , 1 4 5}} \mathbf{9}_{\mathbf{3 4 , 6 5 1}} \mathbf{0}_{\mathbf{4 5 , 2 1 3}} \mathbf{A}_{\mathbf{6 5 , 1 3 4}} \mathbf{B}_{\mathbf{6 1 , 5 3 4}}}{\mathbf{7}_{\mathbf{1 2 , 5 4 6}} \mathbf{8}_{\mathbf{3 2 , 4 6 1}} \mathbf{9}_{\mathbf{3 4 , 2 6 1}} \mathbf{0}_{\mathbf{5 4 , 6 1 2}} \mathbf{A}_{\mathbf{5 6 , 4 1 2}}, \mathbf{B}_{\mathbf{6 1 , 3 2 4}}} \\
& =\frac{\left[\mathbf{3} A_{1}\right]\left[\mathbf{3} A_{2}\right]}{\left[\mathbf{6} A_{1}\right]\left[\mathbf{6} A_{2}\right]} \frac{\left[\mathbf{5} B_{1}\right]\left[\mathbf{5} B_{2}\right]}{\left[\mathbf{6} B_{1}\right]\left[\mathbf{6} B_{2}\right]} \frac{\left[\mathbf{5} C_{1}\right]\left[\mathbf{5} C_{2}\right]}{\left[\mathbf{2} C_{1}\right]\left[\mathbf{2} C_{2}\right]} \frac{\left[\mathbf{3} D_{1}\right]\left[\mathbf{3} D_{2}\right]}{\left[\mathbf{6} D_{1}\right]\left[\mathbf{6} D_{2}\right]} \frac{\left[\mathbf{3} E_{1}\right]\left[\mathbf{3} E_{2}\right]}{\left[\mathbf{2} E_{1}\right]\left[\mathbf{2} E_{2}\right]} \frac{\left[\mathbf{5} F_{1}\right]\left[\mathbf{5} F_{2}\right]}{\left[\mathbf{2} F_{1}\right]\left[\mathbf{2} F_{2}\right]},
\end{aligned}
$$

where $A_{1}, A_{2}$ is a partition of $\mathbf{1}, \mathbf{2}, \mathbf{4}, \mathbf{5}$ into two parts, so is $D_{1}, D_{2}$; and $C_{1}, C_{2}$ is a partition of $\mathbf{1}, \mathbf{3}, \mathbf{4}, \mathbf{6}$ into two parts, so is $F_{1}, F_{2}$; and $B_{1}, B_{2}$ is a partition of $\mathbf{1}, \mathbf{2}, \mathbf{3}, \mathbf{4}$ into two parts; and $E_{1}, E_{2}$ is a partition of $\mathbf{1}, \mathbf{4}, \mathbf{5}, \mathbf{6}$ into two parts.

In the above proof, the partitions are automatically chosen according to the representations of the poles. For example,

$$
\frac{7_{21,345}}{7_{12,546}}=-\frac{[123][453]}{[126][456]}
$$

because the partition $\mathbf{1 2 , 4 5}$ is already provided by the representations.

(5) The fourth step is a conic transformation [11].

Let $p$ be a bracket polynomial which is neither contractible nor factorable in the polynomial ring of brackets. For any six conic points $\mathbf{A}, \mathbf{B}, \mathbf{C}, \mathbf{D}, \mathbf{X}, \mathbf{Y}$ in $p$, if the transformation

$$
[\mathbf{X A B}][\mathbf{X C D}][\mathbf{Y A C}][\mathbf{Y B D}]=[\mathbf{X A C}][\mathbf{X B D}][\mathbf{Y A B}][\mathbf{Y C D}]
$$

either reduces the number of terms of $p$, or makes it factorable in the polynomial ring of brackets, or makes it contractible, the transformation is called a conic transformation.

In this example, the transformation is carried out to

$$
p=[\mathbf{1 2 6}]^{4}[\mathbf{1 3 5}]^{4}[\mathbf{2 3 4}]^{3}[\mathbf{2 4 5}][\mathbf{3 4 6}][\mathbf{4 5 6}]^{3}-[\mathbf{1 2 3}]^{3}[\mathbf{1 2 5}][\mathbf{1 3 6}][\mathbf{1 5 6}]^{3}[\mathbf{2 4 6}]^{4}[\mathbf{3 4 5}]^{4}
$$

as follows: Let $T$ be the first term. Its square-free factors are

$$
p^{\prime}=[\mathbf{1 2 6}][\mathbf{1 3 5}][234][\mathbf{2 4 5}][346][456] .
$$


The conic points with their degrees in $p^{\prime}$ are $\mathbf{1}^{2}, \mathbf{2}^{3}, \mathbf{3}^{3}, \mathbf{4}^{4}, \mathbf{5}^{3}, \mathbf{6}^{3}$.

Since $\mathbf{1}$ has the lowest degree, we select in $p^{\prime}$ those brackets containing $\mathbf{1}$. They are $b(\mathbf{1})=[\mathbf{1 2 6}][\mathbf{1 3 5}]$. The remainder of $b(\mathbf{1})$ in $p^{\prime}$ is $\bar{b}(\mathbf{1})=[\mathbf{2 3 4}][\mathbf{2 4 5}][\mathbf{3 4 6}][\mathbf{4 5 6}]$.

4 is the only conic point not in $b(\mathbf{1})$. Both $[\mathbf{2 3 4}][\mathbf{4 5 6}]$ and $[\mathbf{2 4 5}][\mathbf{3 4 6}]$ are in $\bar{b}(\mathbf{1})$. If we choose $[\mathbf{2 3 4}][\mathbf{4 5 6}]$, then the transformation

$$
([\mathbf{1 2 6}][\mathbf{1 3 5}][\mathbf{2 3 4}][\mathbf{4 5 6}])^{3}=([\mathbf{1 2 3}][\mathbf{1 5 6}][\mathbf{2 4 6}][345])^{3}
$$

changes $T$ to $[\mathbf{1 2 3}]^{3}[\mathbf{1 2 6}][\mathbf{1 3 5}][\mathbf{1 5 6}]^{3}[\mathbf{2 4 5}][\mathbf{2 4 6}]^{3}[\mathbf{3 4 6}][\mathbf{3 4 5}]^{3}$, which has common factors $[\mathbf{1 2 3}]^{3}[\mathbf{1 5 6}]^{3}[\mathbf{2 4 6}]^{3}[\mathbf{3 4 5}]^{3}$ with the second term of $p$. After removing the factors, we get

$$
p=[\mathbf{1 2 6}][135][245][346]-[125][136][246][345] .
$$

Another conic transformation changes $p$ to 0 . If we choose the other pair $[\mathbf{2 4 5}][\mathbf{3 4 6}]$, then the order between the above two transformations is reversed.

(6) The additional nondegeneracy conditions include all the point-conic nondegeneracy conditions required by the transformation rules, and the denominator obtained after the transformation rules are applied.

(7) In (29), there are a lot of freedoms in choosing the partitions - we do not need to follow the partitions provided by the representations. The following is the unique choice of partitions for the result to be a bracket polynomial instead of a rational bracket polynomial.

$$
\begin{aligned}
& \frac{\left[\mathbf{3} A_{1}\right]\left[\mathbf{3} A_{2}\right]}{\left[\mathbf{6} A_{1}\right]\left[\mathbf{6} A_{2}\right]} \frac{\left[\mathbf{3} D_{1}\right]\left[\mathbf{3} D_{2}\right]}{\left[\mathbf{6} D_{1}\right]\left[\mathbf{6} D_{2}\right]} \frac{\left[\mathbf{5} C_{1}\right]\left[\mathbf{5} C_{2}\right]}{\left[\mathbf{2} C_{1}\right]\left[\mathbf{2} C_{2}\right]} \frac{\left[\mathbf{5} F_{1}\right]\left[\mathbf{5} F_{2}\right]}{\left[\mathbf{2} F_{1}\right]\left[\mathbf{2} F_{2}\right]} \frac{\left[\mathbf{5} B_{1}\right]\left[\mathbf{5} B_{2}\right]}{\left[\mathbf{6} B_{1}\right]\left[\mathbf{6} B_{2}\right]} \frac{\left[\mathbf{3} E_{1}\right]\left[\mathbf{3} E_{2}\right]}{\left[\mathbf{2} E_{1}\right]\left[\mathbf{2} E_{2}\right]} \\
& =\frac{[\mathbf{3 1 5}][\mathbf{3 2 4}]}{[\mathbf{6 1 5}][\mathbf{6 2 4}]} \frac{[\mathbf{3 1 4}][\mathbf{3 2 5}]}{[\mathbf{6 1 4}][\mathbf{6 2 5}]} \frac{[\mathbf{5 1 3}][\mathbf{5 4 6}]}{[\mathbf{2 1 3}][\mathbf{2 4 6}]} \frac{[\mathbf{5 1 4}][\mathbf{5 3 6}]}{[\mathbf{2 1 4}][\mathbf{2 3 6}]} \frac{[\mathbf{5 1 3}][\mathbf{5 2 4}]}{[\mathbf{6 1 3}][\mathbf{6 2 4}]} \frac{[\mathbf{3 1 5}][\mathbf{3 4 6}]}{[\mathbf{2 1 5}][\mathbf{2 4 6}]}
\end{aligned}
$$

How is this unique choice obtained? After eliminating $\mathbf{7}, \mathbf{8}, \mathbf{9}, \mathbf{0}, \mathbf{A}, \mathbf{B}$, we get

$$
\begin{aligned}
\frac{[\mathbf{1 2 4}][\mathbf{1 2 5}][\mathbf{1 3 4}][\mathbf{1 3 6}][\mathbf{1 4 5}][\mathbf{1 4 6}][\mathbf{2 4 5}][\mathbf{3 4 6}]}{} & \left\{[\mathbf{1 3 4}][\mathbf{1 3 5}]^{4}[\mathbf{1 4 5}][\mathbf{2 3 4}][\mathbf{2 3 5}][\mathbf{2 4 5}][\mathbf{3 4 6}][\mathbf{3 5 6}][\mathbf{4 5 6}]\right. \\
& \left.-[\mathbf{1 2 3}][\mathbf{1 2 4}][\mathbf{1 2 5}][\mathbf{1 3 6}][\mathbf{1 4 6}][\mathbf{1 5 6}][\mathbf{2 3 6}][\mathbf{2 4 6}]^{4}[\mathbf{2 5 6}] k\right\} .
\end{aligned}
$$

Denote the bracket coefficient of $k$ in $(35)$ by $c$, denote the first term by $c^{\prime}$.

There are two approaches to find the best representation of $k$. The first approach is to consider the brackets in $c$ occurring in a unique manner in the denominator of $k$. There are 4 such brackets:

$$
\begin{aligned}
& {[123] \longrightarrow \frac{[153]}{[123]} * \frac{[546]}{[246]} \quad \text { of type } \frac{5}{2}} \\
& {[\mathbf{1 2 5}] \longrightarrow \frac{[\mathbf{1 3 5}]}{[\mathbf{1 2 5}]} * \frac{[346]}{[\mathbf{2 4 6}]} \quad \text { of type } \frac{3}{\mathbf{2}}} \\
& {[\mathbf{1 3 6}] \longrightarrow \frac{[\mathbf{1 3 5}]}{[\mathbf{1 3 6}]} * \frac{[\mathbf{2 4 5}]}{[\mathbf{2 4 6}]} \quad \text { of type } \frac{\mathbf{5}}{\mathbf{6}}} \\
& {[156] \longrightarrow \frac{[153]}{[156]} * \frac{[243]}{[246]} \quad \text { of type } \frac{3}{6}}
\end{aligned}
$$


Then the other 4 ratios in $k$ can be uniquely determined.

The second approach is to consider the ratio $k^{\prime}=c^{\prime} / c$ and prove that it is a representation of $k$. Start with the bracket with the highest degree in the denominator (or numerator) of $k^{\prime}$. Distribute it among the brackets in the numerator (or denominator) of $k^{\prime}$ to make ratios of types $\mathbf{3} / \mathbf{6}, \mathbf{5} / \mathbf{2}, \mathbf{5} / \mathbf{6}, \mathbf{3} / \mathbf{2}$. Then proceed to the brackets of lower degree in the denominator (or numerator). In this example, starting with $[\mathbf{2 4 6}]^{4}$ (or $[\mathbf{1 3 5}]^{4}$ ), we get the same pairing as in (36).

(8) By reducing the number of changes in the representations, we can reduce the number of additional nondegeneracy conditions. For example, according to (28), we only need to change two representations instead of six:

$$
\begin{aligned}
& {[9 \mathrm{BC}]=\left[\begin{array}{llll}
\mathbf{7}_{21,345} & \mathbf{8}_{23,145} & \mathbf{0}_{45,261}
\end{array}\right]\left[\begin{array}{lll}
\mathbf{9}_{34,612} & \mathrm{~A}_{65,134} & \mathrm{~B}_{61,534}
\end{array}\right]} \\
& -\left[\begin{array}{lll}
7_{12,534} & 0_{54,612} & A_{56,412}
\end{array}\right]\left[\begin{array}{llll}
8_{32,461} & 9_{34,261} & B_{61,345}
\end{array}\right] \frac{8_{23,145}}{\mathbf{A}_{65,134}} \\
& =16 \underline{[\mathbf{1 2 4}][\mathbf{1 2 5}][\mathbf{1 3 4}]^{2}[\mathbf{1 3 5}]^{2}[\mathbf{1 3 6}][\mathbf{1 4 5}]^{2}[\mathbf{1 4 6}][\mathbf{2 3 5}][\mathbf{2 4 5}][\mathbf{2 4 6}]^{2}} \\
& {[\mathbf{3 4 6}][356]\{[123][156][245][346]-[125][136][234][456]\}} \\
& \stackrel{\text { conic }}{=} 0 \text {. }
\end{aligned}
$$

Additional nondegeneracy conditions: $\exists \mathbf{1 2 3 4 5}, \exists \mathbf{1 2 3 4 6}, \exists \mathbf{1 2 4 5 6}, \exists \mathbf{1 3 4 5 6}$.

\section{References}

[1] M. Barnabei, A. Brini, G.-C. Rota. On the Exterior Calculus of Invariant Theory. J. Algebra, 1985, 96: 120-160.

[2] R. Bix. Conics and Cubics. Springer, 1998.

[3] J. Bokowski and B. Sturmfels. Computational Synthetic Geometry. LNM 1355, Springer, Berlin, Heidelberg. 1989.

[4] S. C. Chou. Mechanical Geometry Theorem Proving. D. Reidel, Dordrecht, 1988.

[5] S. C. Chou, X. S. Gao and J. Z. Zhang. Machine Proofs in Geometry-Automated Production of Readable Proofs for Geometric Theorems. World Scientific, Singapore, 1994.

[6] H. Crapo and J. Richter-Gebert. Automatic Proving of Geometric Theorems, in: Invariant Methods in Discrete and Computational Geometry, N. White (ed.), Kluwer Academic Publishers, 1994, 107-139.

[7] P. Doubilet, G. C. Rota and J. Stein. On the Foundations of Combinatorial Theory IX: Combinatorial Methods in Invariant Theory, Stud. Appl. Math., 1974, 57, 185-216.

[8] X. S. Gao and D. Wang. Mathematics Mechanization and Applications. Academic Press, London, 2000.

[9] H. Li and Y. Wu. Automated Theorem Proving with Bracket Algebra in Projective Geometry. In Computer Mathematics, X. S. Gao and D. Wang (eds.), World Scientific, Singapore 2000, pp. $120-129$.

[10] H. Li and Y. Wu. Automated Short Proof Generation for Projective Geometric Theorems with Cayley and Bracket Algebras, I. Incidence Geometry. Accepted by J. of Symbolic Computation. Also available at http://www.mmrc.iss.ac.cn/annal/li1.ps. 
[11] H. Li and Y. Wu. Automated Short Proof Generation for Projective Geometric Theorems with Cayley and Bracket Algebras, I. Conic Geometry. Accepted by J. of Symbolic Computation. Also available at http://www.mmrc.iss.ac.cn/annal/li2.ps.

[12] H. Li and Y. Wu. Automated Theorem Proving in Affine Geometry with Cayley and Bracket Algebras, preprint.

[13] B. Mourrain. New Aspects of Geometrical Calculus with Invariants. Advances in Mathematics, to appear. Also in MEGA 91, 1991.

[14] J. Richter-Gebert. Mechanical Theorem Proving in Projective Geometry. Annals of Math. and Artificial Intelligence, 1995, 13, 159-171.

[15] J. Richter-Gebert. Realization Spaces of Polytopes. LNM 1643, Springer, Berlin, Heidelberg. 1996.

[16] B. Sturmfels. Computational Algebraic Geometry of Projective Configurations. J. Symbolic Computation, 1991, 11, 595-618.

[17] B. Sturmfels. Algorithms in Invariant Theory. Springer, New York, 1993.

[18] B. Sturmfels and W. Whiteley. On the Synthetic Factorization of Homogeneous Invariants. J. Symbolic Computation, 1991, 11, 439-454.

[19] D. Wang. Elimination Methods. Springer, Wien New York, 2001.

[20] N. White. The Bracket Ring of Combinatorial Geometry I. Trans. Amer. Math. Soc., 1975, 202, 79-103.

[21] N. White. Multilinear Cayley Factorization. J. Symb. Comput., 1991, 11, 421-438.

[22] T. McMillan and N. White. The Dotted Straightening Algorithm. J. Symb. Comput., 1991, 11: 471-482.

[23] W. Whiteley. Invariant Computations for Analytic Projective Geometry. J. Symb. Comput., 1991, 11: 549-578.

[24] W. T. Wu. Mathematics Mechanization. Science Press/Kluwer Academic, Beijing 2000. 